\title{
Théorie des catastrophes et dynamisme sémantique
}

\begin{abstract}
The article focuses on the potential import of catastrophe theory in the study of word semantics. Thus the lexical content of the French adjective terrible is analyzed within the dynamic geometrical frame-work of the catastrophe theoretic schemata in order to apprehend, on the one hand, the continuum of similar meanings between the two senses (dreadful/formidable) of the word and, on the other hand, the discontinous reversal between the two opposite values.
\end{abstract}

\section{Introduction}

Depuis bien déjà une dizaine d'années, la recherche en matière de linguistique s'est vue enrichie d'une méthodologie nouvelle relevant de la dite théorie des catastrophes comme approche géométrico-topologique de la structure et de la forme ${ }^{1}$. Nonobstant les réactions jusqu'à présent

1 Une première présentation de la théorie des catastrophes fait surface en 1972 lors de la publication, longuement attendue, de l'ouvrage Stabilité structurelle et morphogénèse du mathématicien René Thom. Le livre marque un tournant scientifique décisif, soit, au sens de Kuhn, la constitution d'un nouveau paradigme, sous la forme d'une morphologie générale visant à formaliser, dans un cadre géométrico-topologique, les problèmes de la morphogénèse, de la création et de la destruction de formes, de leur stabilité structurelle fondatrice et changements brusques ou catastrophiques, indépendamment du support - du dit espacesubstrat -matériel ou immatériel, animé ou inanimé. La T.C. s'applique de la sorte aussi bien à l'étude, en physique, des singularités des fronts d'onde ou, en biologie, du développement embryologique qu'à l'étude, en linguistique, par exemple, du dynamisme sémantique. En tous 
généralement dénégatrices du côté des sciences du langage à l'égard d'une visée stratégique d'inspiration mathématique, de nombreux apports d'ordre phonologique, syntaxique et sémantique ont attesté et affermi la pertinence de la démarche catastrophiste dans l'étude des phénomènes signifiants 2 .

Le présent travail se propose de renouer avec la stratégie de la théorie des catastrophes (T.C.) pour donner en exemple de son opérationnalité pratique, l'analyse d'un phénomène de dynamisme sémantique. En l'occurence, le dynamisme sous-jacent au sens antinomique de l'adjectif "terrible".

Toute appréciation tant soit peu profonde des opérations de la T.C. suppose d'évidence une connaissance minimale des mathématiques qu'elles mettent en œuvre. Or, dans ce qui suit, les notions de géométrie et de topologie différentielles, constitutives de la T.C., restent le plus souvent implicitées, l'essentiel ici n'étant pas d'introduire aux propositions et aux théorèmes catastrophistes, éminemment sophistiqués, mais d'illustrer une application hautement opératoire de leur portée analytique ${ }^{3}$.

\section{L'exemple de "terrible"}

Soit, donc, l'adjectif français "terrible"4. Selon le dictionnaire Robert, "terrible" peut avoir deux sens différents, antinomiques. Un sens négatif : Qui inspire de la terreur, et un sens positif, synonyme de extraordinaire, grand. Aussi l'énoncé "c'est terrible" est-il ambigu. Comment "terrible" : effroyable ou formidable? Terrifiant ou grand? Le sens flotte, instable parce que non-

domaines, la même conceptualisation, la même méthodologie et la même modélisation, président à l'analyse.

2 Voir par exemple Petitot (1982), (1985a), (1985b), (1991); Thom (1972), (1974), (1980); Whildgen (1981), (1982).

3 Pour la précision des mathématiques, cf. par exemple Saunders (1980), Thom (1972), (1980).

L'exemple s'inspire de Poston (1987). 
marqué, détâché de tout ancrage de la signification, c'est-à-dire de l'énonciation et/ou du contexte. La constitution du sens d'un énoncé, le contenu propositionnel, est, comme y aura bien insisté la pragmatique, déterminée d'un côté par l'énonciation (l'intonation, la modulation, le débit, la mimique, la gesticulation, etc.), et d'un autre côté par le contexte (textuel et spatio-temporel). Aussi la production du sens est-elle un procès dynamique, et plutôt qu'en termes de structures, c'est en termes de procédure qu'il faudra penser la signification.

Nous pouvons ainsi, à titre d'exemple d'un dynamisme sémantique, analyser le devenir du sens d'un énoncé en tant que de fonction d'un procès de stabilisation du signifié par l'énonciation et le contexte.

Pour nous en tenir à notre exemple, la conceptualisation de "terrible" délimite le potentiel du sens de l'adjectif en positionnant l'expression dans un espace sémantique exfolié en un spectre discret de significations paramétrisées respectivement par leur degré de positivité et d'intensité. Soit par exemple:

Fig. 1

Suivant le ton ou l'environnement situationnel, l'expression "terrible", d'un côté, se rapporte au sens univoque de "épouvantable" ou bien, de l'autre 
côté, au sens également univoque de "super", alors qu'elle se distingue, par le degré de son intensité, du sens d'expressions moins fortes telles que "vache", "pas mal" ou "chouette". Or cette bimodalité du potentiel de sens, savoir les significations négatives et positives de l'expression "terrible" en tant que scindées, d'une part, de façon discontinuiste ("terribleépouvantable"/"terrible-super"), mais aussi, et d'autre part, en tant que reliées de façon continuiste (par des valeurs lexicales de positivité et d'intensité graduées interposées: "terrible"-"épouvantable"-"vache"-"pas mal""chouette"-"super" -"terrible"), se laisse penser précisément selon le théorème de Thom des systèmes dynamiques, structurellement stables 5 , décrits par deux coordonnés sur un espace de configuration (une variété différentiable).

Soit $\mathrm{u}=$ intensité et $\mathrm{v}=$ positivité deux variables externes dites de contrôle et soit $\mathrm{x}=$ conceptualité une variable interne dite d'état. $\mathrm{u}$ paramétrise l'expression en tant que plus ou moins "forte". v paramétrise l'expression en tant que plus ou moins positive ou négative. $\mathrm{x}$ alors conceptualise l'expression variant en fonction du degré d'intensité et de positivité. Les valeurs en baisse de $\mathrm{x}$ correspondent aux contenus de désapprobation, les valeurs à la hausse aux contenus d'approbation.. L'ensemble $\mathrm{M}$ des états $\mathrm{x}$ est supposé constituer un espace sémantique de valeurs conceptualisées, relatives, sans solution de continuité, aux contenus stables, dits "attracteurs", définis par projection conceptuelle de l'expression linguistique dans un espace de représentation 6 .

Ainsi, par exemple, l'expression "pas mal", dite sur un ton enthousiasmé, sortirait-elle de sa conceptualisation habituelle, plutôt neutre, pour être capturée par un attracteur plus positivisé, au voisinage, si l'on veut, du

5 La notion de stabilité structurelle constitue un principe de raison géométricotopologique suffisante de la T.C.: "La stabilité structurelle "cause" les morphologies dans la mesure où celles-ci ne sont que les "solutions" au problème qu'elle pose" (Petitot 1977, 11). "Quelque chose ne peut exister que s'il est structurellement stable, c'est-à-dire s'il résiste aux perturbations "infinitésimales" dues à son environnement" (ibid., 51).

6 Nous ne parlerons pas ici des problèmes délicats de la dynamique neuronale - dite aussi"connexioniste" - de la cognition. Pour plus de précision, voir Petitot (1991). 
contenu de sens de "chouette" (Robert: qui est digne d'admiration, d'éloge). $\mathrm{Ou}$, de même, l'expression "vache", prononcée sur un ton énergique, vigoureux, figurerait-elle au voisinage d'un attracteur plus intense, tel celui de "fâcheux" (Robert: qui est pour qqn une cause de déplaisir ou de souffrance) ou de "contrariant" (Robert: qui contrarie, c'est-à-dire : qui rend inquiet, mal à l'aise), lesquels ne figurent pas dans notre relevé mais qui d'évidence y auront leur place. Effectivement, à toute expression valorisante correspond(ent) un (ou des) "bassin(s)" d'attracteur(s). Cependant, l'essentiel ici n'est pas (et de loin) le repérage exhaustif des domaines de sens définis sur M, ni non plus, d'ailleurs, l'exactitude de la disposition topologique relative (la différentiation) des bassins associés à différents attracteurs. Seuls nous occupent des principes méthodologiques. Aussi nous contentons-nous d'une simple configuration intuitive de contenus de sens exemplaires. (Une localisation précise des attracteurs et la répartition exacte en bassins, ce qui n'est évidemment en rien exclu, demanderaient des expériences pratiques à l'aide d'informants.)

Maintenant, suivant le théorème de Thom des systèmes dynamiques assujettis à deux variables de contrôle, la topologie la plus simple des attracteurs structurellement stables constitue la géométrie de la catastrophe structurellement stable, nommée le cusp ${ }^{7}$. A déployer la dite "singularité organisatrice" de cette géométrie, nous obtiendrons (par application différentiable) un espace plié aux contraintes générales de la stabilité structurelle :

7 C'est le nom d'une surface dont la topologie s'écrit, en supposant la variété différentiable $\mathrm{M}=\_\mathrm{V} / \mathrm{X}_{\mathrm{X}}=0$ :

$$
\begin{aligned}
\mathrm{V} & =\mathrm{x}^{4} \mathrm{ux}^{2} \_\mathrm{vx} \\
\mathrm{V}_{-} / \mathrm{x}^{3} & =4 \mathrm{x}^{3}{ }_{-} \mathrm{ux}_{-} \mathrm{v}
\end{aligned}
$$

Mais laissons de côté ici les mathématiques présidant au "déploiement universel" de la singularité organisatrice $x^{4}$ ainsi qu'à son "application différentiable", structurellement stable. Le lecteur intéressé pourra se reporter aux ouvrages cités à la note 3 . 
Fig. 2

Le modèle montre comment l'espace $M$ des états $\mathrm{x}$ dépend de deux contrôles (u, v). Pour u > 0, M se scinde en deux surfaces de types de qualification opposés, respectivement en une surface des états $\mathrm{x}$ de valeurs hautes et en une surface des états $\mathrm{x}$ de valeurs basses. En tant que modèle d'un dynamisme sémantique, nous pourrons y marquer nos conceptualisations exemplaires (fig. 3). On s'imaginera chaque expression conceptualisée, non pas par un seul point, mais par un nuage de points, selon les divers modes de son actualisation énonciative ou contextuelle possibles. Certains points marquent ainsi les voisinages, sinon l'identité, de plusieurs expressions (synonymie) : 
Fig 3

Pour ce qui concerne l'exemple de "terrible", nous voyons l'emplacement de son potentiel intense dans le domaine de la scission de M, c'est-à-dire dans le champ fonctionnel des points à double valeur. La conceptualité (i.e. le sens signifié: grand ou terrifiant) sera actualisée par le contexte (textuel ou situationnel) et/ou l'énonciation: par leur charge positive ou négative, déterminant de la positivité $\mathrm{v}$ des expressions, donc de la valeur conceptuelle (d'approbation ou de désapprobation). Ainsi une intonation enthousiaste d'emportement, ou bien, à l'inverse, une tonalité tremblant d'épouvante rendront-elles évident le contenu de sens de "terrible". De même, un contexte positif ou négatif aura un effet de désambiguïsation de l'expression. Dans des contextes neutres, indécidables, la conceptualisation dépend de la seule énonciation. Tout comme elle dépend, pour des intonations neutres, indécidables, du seul contexte. Si ni l'énonciation ni le contexte ne sont marqués (e.g. une phrase écrite, isolée), il n'y aura pas moyen de déterminer le sens de "terrible", L'expression serait aussi bien positive que négative. L'énonciation et le contexte sont bien alors ce qui stabilise le sens signifié comme valeur conceptuelle approbatrice ou désapprobatrice: ils constituent la dynamique même du procès de signification.

Pour $\mathrm{u}>0$, la trajectoire de $\mathrm{f}_{(\mathrm{v})}(\mathrm{x})$, passant d'un contexte ou d'une énonciation de type négatif à un contexte ou énonciation de type positif, décrira un changement de sens de "terrible" (effroyable $\longrightarrow$ formidable) lors 
de la traversée d'un point pli de la surface inférieure, tandis qu'en sens inverse le changement (la catastrophe) se produira lors de la traversée d'un point pli de la surface supérieure 8 :

Fig 4

"Terrible" n'est plus dès lors une pure fonction linéaire de la positivité du contexte ou de l'énonciation. Le mot peut, jusqu'à un certain degré (qu'on appelle catastrophique) exprimer un sens d'approbation sur le mode (énonciateur ou contextuel) négatif, et inversement, jusqu'à un certain point, exprimer un sens de désapprobation sur un mode positif! L'expression ironique, le jeu de mot, et bien d'autres emplois ludiques ou détournées du langage, jouant sur le double sens, pourraient avec profit être analysés dans cette optique de la T.C 9 .

Plutôt que d'une approche linguistique traditionnelle, déterminant la valeur du sens en termes de structures sémantiques comme fonction directe $\mathrm{du}$ contexte et de l'énonciation, le dynamisme de la modélisation catastrophiste permet une analyse fonctionnelle plus nuancée du sens sur fond de chemins génériques (de trajectoires significatives) dans l'espace des états de conceptualisation. Ainsi de même, par exemple, le devenir du sens en tant que d'effet du seul degré d'intensité (i.e. de l'évolution de $\mathrm{f}_{(\mathrm{u})}(\mathrm{x})$ pour des

8 On parle ici de "cycle d'hystérésis".

9 Cf. Lund (1992b). 
valeurs v non-marquées). Selon le Dictionnaire du français contemporain, l'expression "comme ci comme ça" s'articule d'une position de positivité et d'intensité neutre (au sens propre, originaire, du terme: en latin "ne...uter" veut dire ni...ni): Ni bien ni mal, moyennement. Or, constat trivial, cette neutralité, voilà justement ce qui ne peut être intensifiée sauf à se perdre et à prendre parti, c'est-à-dire sauf à exprimer soit l'approbation, soit la désapprobation. C'est ce qu'illustre assez le modèle du cusp, où l'état neutre (ni...ni), relativement à une progression intense du dire, correspondraient au "strate" intermédiaire inaccessible pour se voir contraint de diverger en des valeurs conceptuelles bimodalisées (soit...soit) 10 :

Fig. 5

$\overline{10}$ A l'encontre des strates supérieure et inférieure dont la stabilité structurelle est constituée par les minima d'une fonction potentielle $\mathrm{f}$, la strate intermédiaire est constituée par les maxima structurellement instables. Elle représente comme telle des états mathématiquement invraisemblables. 
Intensifié (par la tonalité de la voix, la mimique, l'attitude du corps..., soit par la situation, l'à-propos, l'ambiance régnante...), le sens signifié de "comme ci comme ça" sera toujours d'un côté ou de l'autre, pour ou contre, jamais entre-les-deux, jamais neutre.

\section{Perspectives}

Avec l'apport des techniques de formalisation et de modélisation de la T.C. en matière linguistique, nous disposons d'une méthode d'observation et d'analyse fine de situations de langage. Et non seulement pour l'élucidation du sémantisme dynamique. Effectivement, l'appréhension catastrophique de la phénoménologie du langage porte sur toute une gamme de problématiques sémio-linguistiques.

Pour passer en revue quelques-unes des plus significatives, citons à titre d'exemple les travaux de Jean Petitot sur les catastrophes de la parole ${ }^{11}$, opérant en tant qu'au fondement du rapport de la phonologie et de la phonétique comme l'articulation morphologique de leurs paradigmes disparates, du formalisme structuraliste de l'un et de la substantialité psychoacoustique de l'autre. Mais surtout les recherches de Petitot sur ce qu'il appelle la "physique du sens" et qui portent sur le rapport du langage au monde, soit à l'objectivité phénoménologique à laquelle il renvoie comme à sa raison de dire, mais comme ce lien même à l'être auquel il lie. C'est là dire la conformité l'un à l'autre des deux ordres langagier et mondain, linguistique et ontologique, par l'isomorphisme de leurs relations morphologiques commune de reproduction l'un en l'autre.

Il n'y a pas d'un côté le langage et de l'autre (en face) le monde, la réalité. En se rapportant à l'être, le langage le rapporte en lui: en tant qu'être même du rapport à l'être ${ }^{12}$.

\footnotetext{
11 Petitot $(1985 a)$

12 Cf. Ouellet (1987).
} 
Or, le sens linguistique relève de cette identité formelle, de cette isomorphisme, ou co-formalité, qui d'ores et déjà ouvre la voie à un sémantisme formel de l'être du rapport, soit à une schématisation des morphologies relationnelles ontologiquement déterminante. En quoi la physique du sens de Petitot rejoint les travaux de morphogenèse en linguistique de René Thom portant justement sur l'objectivité des formes de langage.

De quelle nature sont ces formes relationnelles (propositionnelles et objectives)? Quel en est le principe régulateur? Participant de quelle logique? Comment, enfin, les schématiser? Par la voie des morphologies dites "archétypes", les "graphes actantiels" élaborés, sur la base d'a priori de la stabilité structurelle des schèmes catastrophiques élémentaires, en fonction d'une géométrie de la position, soit d'une configuration positionnelle d'actants (de rôles sémantiques) dans un espace-temps abstrait, commun au fait langagier et au fait référentiel 13 .

Mais déjà cela nous entraîne trop loin que de poursuivre cette ontogenèse des formes signifiantes. De même, et pour conclure, contentons-nous de renvoyer simplement, pour des approfondissements d'analyse des archétypes thomiens, aux travaux de sémantique appliquée de Wolfgang Wildgen ${ }^{14}$. S'il reste encore pour l'instant nombre de problèmes de méthodologie à clarifier (de l'ordre de la topologisation, soit de la paramétrisation et, par là, de la schématisation), l'apport du formalisme de la T.C. en lexicologie, mais cela vaut pour son investigation de la linguistique dans son ensemble, paraît cependant hautement prometteur. Ceci notamment parce que la T.C. propose des schèmes d'intelligibité, de description et d'explication de phénomènes qui, autrement, seraient souvent très délicats à approcher et à manier.

\section{Bibliographie}

\footnotetext{
13 Pour une brève présentation de ces archétypes sémantiques, voir Lund (1992a).

14 Voir notamment Wildgen (1981); (1982).
} 
Lund, Steffen Nordahl (1992a): Katastrofeteori og semantik. For en dynamisk og ontologisk fundering af sprogbeskrivelsen. In: Merino 11, $21 \mathrm{~s}$.

Lund, Steffen Nordahl Lund (1992b): Ordspillets dynamik. In: Almen semiotik 5 113-117.

Ouellet, Pierre (1987): La nouvelle Aufklärung: Une physique du sens. In: Critique 481-482, 577-597.

Petitot, Jean (1977): Cartographie élémentaire. In: Mathématiques et Sciences humaines 59, 725.

Petitot, Jean (1982): Sur la signification linguistique de la théorie des catastrophes. In: Mathématiques et Sciences humaines 79, 37-74.

Petitot, Jean (1985a): Les catastrophes de la parole. Paris: Maloine.

Petitot, Jean (1985b): Morphogenèse du Sens I. Paris: PUF.

Petitot, Jean: (1991): Syntaxe topologique et grammaire cognitive. In: Langage 103, 97-127.

Poston, T. (1987): “Mister! Your back wheel's going round!”. In: Thomas T. Ballmer and Wolfang Wildgen (ed.): Process Linguistics. Tübingen: Max Niemeyer Verlag, 11-36.

Saunders, P.T. (1980): An introduction to catastrophe theory. Cambridge: Cambridge University Press.

Thom, René (1972): Stabilité structurelle et morphogénèse. W.A. Benjamin Inc. Deuxième Édition, revue, corrigée et augmentée. Paris: Interédition.

Thom, René (1974): La linguistique, discipline morphologique exemplaire. In: Critique 322, 235-245.

Thom, René (1980): Modèles mathématiques de la morphogénèse. Paris: Christian Bourgois.

Wildgen, Wolfgang (1981): Archetypal Dynamics in Word Semantics: An Application of Catastrophe Theory. In: Hans-Jürgen Eikmeyer and Hannes Rieser (ed.): Words, Worlds, and Contexts. New Approaches in Word Semantics. Berlin, New York: Walter de Gruyter, 234-296.

Wildgen, Wolfgang (1982): Catastrophe Theoretic Semantics. Amsterdam: Benjamin. 